Annuaire suisse de politique de développement

24-1 | 2005

Faits et statistiques 2005

\title{
6. Commerce mondial
}

Natacha Bogorad

\section{OpenEdition}

\section{Journals}

Édition électronique

URL : http://journals.openedition.org/aspd/425

DOI : 10.4000/aspd.425

ISSN : 1663-9669

\section{Éditeur}

Institut de hautes études internationales et du développement

\section{Édition imprimée}

Date de publication : 1 avril 2005

Pagination : 73-90

ISSN : 1660-5934

\section{Référence électronique}

Natacha Bogorad, «6. Commerce mondial », Annuaire suisse de politique de développement [En ligne], 24-1 | 2005, mis en ligne le 02 mars 2010, consulté le 08 septembre 2020. URL : http:// journals.openedition.org/aspd/425; DOI : https://doi.org/10.4000/aspd.425 


\section{Commerce mondial*}

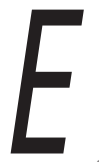

${ }_{N} 2003$, le commerce mondial a repris de la vigueur pour atteindre une croissance de 4,5\%. Cette progression est due en particulier à l'expansion de la demande aux Etats-Unis et en Asie de l'Est. Les prévisions pour 2004 annoncent une croissance qui devrait dépasser les 7\%. Ce sont l'Asie et les économies en transition qui ont enregistré la hausse de leur commerce la plus importante. La part des pays en développement dans la croissance du commerce mondial a encore progressé en 2003.

L'année 2004 a été marquée par la conclusion en juillet d'un accord-cadre à l'OMC. En effet, après d'intenses négociations, les membres de l'OMC ont adopté une décision composée d'une partie générale et de quatre annexes, relatives à l'agriculture, à l'accès aux marchés pour les produits non agricoles, aux services et à la facilitation des échanges. Cet accord permet la relance des négociations du cycle de Doha qui avaient tourné au ralenti suite à l'échec de la Conférence de Cancún en septembre 2003. La prochaine échéance est désormais la Conférence ministérielle de Hongkong, qui se tiendra en décembre 2005.

Cet accord-cadre a donné lieu à des réactions différenciées. La Suisse l'a salué, notamment parce qu'il permet la reprise des négociations. Les ONG suisses et étrangères ont par contre dénoncé un accord déséquilibré et un processus de décision peu transparent.

Le deuxième fait marquant de l'année 2004 réside dans la tenue à São Paulo de la $11^{e}$ Conférence des Nations unies sur le commerce et le développement (CNUCED). Le thème principal était la cohérence entre les visions nationales et internationales du commerce et du développement. La promotion du commerce Sud-Sud a également été mise en avant comme un facteur important de développement. Enfin, le rôle et le mandat de la CNUCED ont été vivement débattus. Les réactions à la CNUCED XI ont été globalement positives, notamment celles des ONG suisses, qui se réjouissent du maintien de l'indépendance critique de la CNUCED.

\subsection{Evolution des relations commerciales}

Le commerce mondial a connu une progression lente au premier semestre 2003, notamment à cause des facteurs temporaires du syndrome respiratoire aigu sévère (SRAS) en Asie de l'Est et de la guerre en Irak. Par contre, il a repris de la vigueur au second semestre. Sur l'année 2003, la croissance mondiale du commerce des marchandises en valeur nominale a atteint $16 \%$ et celle des services $12 \%$. Il convient de relever que la croissance du commerce a été beaucoup plus marquée en valeur nominale (en dollars) qu'en valeur réelle, notamment à cause des fluctuations du taux de change. Ainsi, par exemple, le commerce des marchandises a augmenté de $4,5 \%$ en valeur réelle et de $16 \%$ en valeur nomi-

* Par Natacha Bogorad, lic. IUHEI, IDEAS Centre. 
nale ${ }^{1}$. Cette croissance est due en particulier à l'expansion de la demande aux Etats-Unis et en Asie de l'Est.

Les perspectives pour 2004 annoncent une poursuite de l'accélération de la croissance de l'économie mondiale. Les estimations prévoient une hausse de la production de $3,7 \%$ et du commerce de $7,5 \%$, en termes réels. Cependant, la forte augmentation du prix du pétrole et l'accroissement du déficit américain représentent des risques qui peuvent influencer l'évolution des relations commerciales $^{2}$.

Tableau 15: Exportations mondiales de marchandises et de services commerciaux, 2003 (en milliards de dollars et en pourcentage)

\begin{tabular}{lcrrrr}
\hline & Valeur (mia. de \$) & & \multicolumn{3}{c}{ Variation annuelle (\%) } \\
\cline { 1 - 2 } \cline { 5 - 6 } \cline { 5 - 6 } & $\mathbf{2 0 0 3}$ & $\mathbf{2 0 0 1}$ & $\mathbf{2 0 0 2}$ & $\mathbf{2 0 0 3}$ \\
\hline Marchandises & 7274 & & 4 & & \\
\hline Services commerciaux & 1763 & 0 & 6 & 16 \\
\hline
\end{tabular}

Source: OMC, Rapport sur le commerce mondial 2004, Genève, tableau IA2, p. 7.

Dans le classement des principaux pays exportateurs et importateurs participant au commerce mondial des marchandises de l'Organisation mondiale du commerce (OMC), la Suisse occupe respectivement la $19^{\text {e }}$ et la $18^{\text {e }}$ position $^{3}$. Au niveau des exportations, les premières places sont occupées par l'Allemagne, les Etats-Unis et le Japon, tandis que pour les importations, ce sont les Etats-Unis qui prennent la tête, suivis de l'Allemagne et de la Chine. En termes de services commerciaux, elle se situe au $16^{\mathrm{e}}$ rang des principaux pays exportateurs et au $24^{\mathrm{e}}$ rang des principaux pays importateurs. Ce sont les Etats-Unis, le RoyaumeUni et l'Allemagne qui sont en tête de classement ${ }^{4}$.

\subsubsection{Commerce mondial des marchandises par région}

En 2003, 1'Asie et les économies en transition ont sorti leur épingle du jeu. En effet, l'Asie a connu une croissance de ses exportations et de ses importations de marchandises à deux chiffres aussi bien en valeur nominale $(17 \%$ et $19 \%$ respectivement) qu'en valeur réelle (12\% et $11 \%$ respectivement). Les pays en transition ne sont pas en reste avec une hausse de $28 \%$ de leurs exportations et de $27 \%$ de leurs importations, en valeur nominale, et un accroissement réel de 12 et $11 \%$. L'Amérique latine a connu une hausse de la valeur de ses exportations de marchandises de $9 \%$, mais une progression plus modeste de ses impor-

1 «L'accroissement en volume ou accroissement réel est basé sur les valeurs nominales du commerce corrigées des variations de prix et de taux de change. Ce n'est pas une mesure de la quantité physique des marchandises échangées. » OMC, Rapport sur le commerce mondial 2004, Genève, p. 6.

2 OMC, Statistiques du commerce international 2004, Genève; idem, Rapport sur le commerce mondial 2004, op. cit.

3 La valeur des exportations de marchandises de la Suisse s'élève à 99,4 milliards de dollars, ce qui correspond à une part de 1,3\% des exportations mondiales totales. Celle de ses importations se monte à 95,2 milliards, pour une part de 1,2\% des importations mondiales totales. Pour les services, la valeur des exportations est de 32 milliards de dollars, une part de 1,8\% des exportations mondiales totales, contre 19 milliards de dollars pour les importations, une part de 1,1\% des importations mondiales totales.

4 OMC, Statistiques du commerce international 2004, op. cit., pp. 21 et 23. 
tations de $3 \%$. Au contraire, l'Amérique du Nord a enregistré une croissance de la valeur de ses importations $(8 \%)$ plus forte que celle de ses exportations $(5 \%)$. Quant à l'Europe occidentale, la croissance de ses exportations et de ses importations est restée modeste en taux réels (moins de 2\%), même si en valeur nominale elle s'est élevée à 18 et $19 \%$ respectivement ${ }^{5}$.

Tableau 16: Croissance de la valeur du commerce mondial des marchandises, par région, 2003 (en milliards de dollars et en pourcentage)

\begin{tabular}{|c|c|c|c|c|c|c|c|c|}
\hline \multirow{3}{*}{ Région } & \multicolumn{4}{|c|}{ Exportations } & \multicolumn{4}{|c|}{ Importations } \\
\hline & \multirow{2}{*}{$\begin{array}{l}\text { Valeur } \\
2003\end{array}$} & \multicolumn{3}{|c|}{ Variation annuelle (\%) } & \multirow{2}{*}{$\begin{array}{c}\text { Valeur } \\
2003 \\
\end{array}$} & \multicolumn{3}{|c|}{ Variation annuelle (\%) } \\
\hline & & 1995-00 & 2002 & 2003 & & 1995-00 & 2002 & 2003 \\
\hline Monde & 7294 & 5 & 5 & 16 & 7569 & 5 & 4 & 16 \\
\hline Amérique du Norda & 997 & 6 & -4 & 5 & 1549 & 10 & 2 & 8 \\
\hline Amérique latine & 378 & 10 & 0 & 9 & 366 & 9 & -7 & 3 \\
\hline Europe occidentale & 3145 & 2 & 6 & 18 & 3178 & 3 & 5 & 19 \\
\hline $\begin{array}{l}\text { Union européenne } \\
\text { (Quinze) }\end{array}$ & 2901 & 2 & 6 & 18 & 2920 & 3 & 4 & 19 \\
\hline $\begin{array}{l}\text { Europe centrale et orien- } \\
\text { tale, Etats baltes, CEI }\end{array}$ & 401 & 7 & 10 & 28 & 379 & 4 & 11 & 27 \\
\hline $\begin{array}{l}\text { Europe centrale } \\
\text { et orientale }\end{array}$ & 192 & 8 & 15 & 30 & 226 & 9 & 11 & 27 \\
\hline Fédération de Russie & 134 & 5 & 4 & 25 & 74 & -6 & 12 & 23 \\
\hline Afrique & 173 & 6 & 2 & 23 & 166 & 0 & 2 & 21 \\
\hline Moyen-Orient & 299 & 12 & 2 & 19 & 192 & 4 & 5 & 9 \\
\hline Asie & 1901 & 5 & 8 & 17 & 1739 & 3 & 6 & 19 \\
\hline Japon & 472 & 2 & 3 & 13 & 383 & 2 & -3 & 14 \\
\hline Chine & 438 & 11 & 22 & 34 & 413 & 11 & 21 & 40 \\
\hline $\begin{array}{l}\text { Six pays commerçants } \\
\text { d'Asie de l'Est }\end{array}$ & 688 & 5 & 6 & 14 & 616 & 2 & 4 & 12 \\
\hline
\end{tabular}

Source: OMC, Statistiques du commerce international 2004, Genève, tableau I.3, p. 20.

${ }^{a}$ A l'exclusion du Mexique.

\subsubsection{Place des pays en développement dans le commerce mondial}

En 2003, la croissance du commerce a été générée majoritairement par les pays en développement. En effet, en 2003, ils ont contribué à hauteur de $66 \%$ à l'augmentation du volume des exportations mondiales et à hauteur de $50 \%$ à celle des importations mondiales par rapport à 2002. La croissance du volume du commerce des marchandises des pays en développement s'est élevée à plus de $10 \%$. L'Asie de l'Est et du Sud ont enregistré des valeurs dépassant les $14 \%$. L'Afrique a aussi vu le volume de ses importations et de ses exportations de marchandises augmenter de plus de $20 \%$ en valeur nominale et de pratiquement $8 \%$, en valeur réelle. Cela est dû en particulier à la croissance des exportations des pays exportateurs de pétrole. Quant à l'Amérique latine, elle s'est remise petit à petit de la récession des années précédentes ${ }^{6}$.

Ibid., pp. 8-15.

6 CNUCED, Rapport sur le commerce et le développement 2004, New York; Genève, ONU, 2004, pp. 43-49. 
L'Organisation mondiale du commerce (OMC) a lancé en 2001, à Doha, un nouveau cycle de négociations commerciales, articulé autour du Programme de Doha pour le développement. La dernière Conférence ministérielle de l'OMC, tenue en septembre 2003 à Cancún, devait représenter une étape essentielle de ce processus. Cette conférence s'est cependant soldée par un échec. Les pays membres se sont alors mis d'accord pour relancer le cycle de Doha, dont

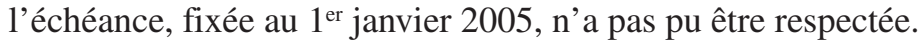

Annuaire 2004, $\mathrm{n}^{\circ}$ 1, chap. 6, point 6.2, pp. 83-96.

\subsubsection{Relance des négociations après Cancún}

De retour à Genève, après la conférence de Cancún, les pays membres se sont mis d'accord pour suspendre les travaux des organes de négociations ${ }^{7}$ et pour mener des consultations informelles sur quatre sujets d'importance: l'agriculture, l'accès aux marchés pour les produits non agricoles, les questions de Singapour (investissement, facilitation des échanges, transparence des marchés publics, politique de la concurrence) et le coton. Lors du dernier Conseil général de l'année, en décembre 2003, le président du Conseil général, Carlos Pérez del Castillo, et le directeur général de l'OMC, Supachai Panitchpakdi, ont fait état de leurs conclusions tirées de ces consultations. ${ }^{8}$. Ils ont souligné que le chemin parcouru depuis Cancún avait été considérable. Ils ont également estimé que les pays membres avaient manifesté leur engagement en faveur du système multilatéral et du Programme de Doha pour le développement. Ils ont donc jugé que les pays membres étaient prêts à reprendre le travail au sein des groupes de négociation. Ils ont par contre mis en avant les nombreuses divergences qui restaient présentes entre les membres et le peu de négociations réelles qui avaient été conduites pendant cette période. La fin de l'année 2003 a donc été marquée par des discussions tournant au ralenti mais également par un état d'esprit constructif pour poursuivre les négociations et réactiver le cycle de Doha.

Au début de l'année 2004, les discussions commerciales ont repris progressivement. Rapidement, des signes d'ouverture pour relancer les négociations sont apparus de la part des deux acteurs de poids de l'OMC. En effet, les Etats-Unis et l'Union européenne ont chacun envoyé une lettre à l'attention des autres membres de l'OMC, contenant leurs propositions pour raviver les négociations". Une réunion du Conseil général en février 2004 a entériné la nomination des nouveaux présidents des organes de l'OMC, ce qui a permis la reprise des travaux de négociation. Pendant les premiers mois de l'année, le climat en faveur

7 Déclarations du président du Conseil général et du directeur général du 14 octobre 2003, $<$ www.wto.org $>>$ Nouvelles $>$ Archives $>$ Nouvelles $>2003$.

8 Statement by the Chairperson of the General Council of 15th December 2003, Statement by DirectorGeneral of 15th December 2003, Remarques finales du président du Conseil général lors de la réunion du Conseil général des 15 et 16 décembre 2003, <www.wto.org > Nouvelles >Archives >Nouvelles $>2003$.

9 Zoellick Embarks on Global Push to Make Strong Progress on Doha Negotiations, 2 August 2004, $<$ www.ustr.gov> Document Library >Press Releases > 2004 Press Releases >February; Lettre du commissaire au Commerce Pascal Lamy et du commissaire à l'Agriculture Franz Fischler, <http://tradeinfo.cec.eu.int/doc\$lib/docs/2004/october/tradoc_119455.pdf>. 
des négociations au sein de l'OMC s'est amélioré et un consensus s'est petit à petit dégagé en faveur de l'objectif d'aboutir à un accord-cadre avant l'été. Pendant toute la période jusqu'au mois de juillet, de nombreuses consultations et réunions se sont tenues entre différents membres de l'OMC, à Genève et en marge de divers sommets ${ }^{10}$. Finalement, après de longues négociations, les membres de l'OMC sont parvenus à un accord-cadre en juillet 2004 (ou ensemble des résultats de juillet).

\subsubsection{Position suisse}

Du point de vue du gouvernement suisse, l'accord-cadre de juillet 2004 est globalement positif. En effet, d'une part, ces résultats donnent un nouvel élan aux négociations du cycle de Doha, après l'échec de la Conférence de Cancún. D'autre part, ils répondent en grande partie aux intérêts de la Suisse. En ce qui concerne l'agriculture, le gouvernement a affirmé que l'accord-cadre, même s'il avançait sur la voie de la libéralisation, tenait «compte des besoins spécifiques de l'agriculture suisse $»^{11}$. L'agriculture représente en effet un thème très sensible pour la Suisse. Ainsi, elle défend ses intérêts au sein du G-10 12 , un groupe de 10 pays qui vise une libéralisation progressive et limitée de ce secteur, ainsi que la prise en compte de ses aspects non commerciaux ${ }^{13}$. Dans le cas de l'accès aux marchés pour les produits non agricoles, la Suisse considère sa libéralisation comme une priorité. Dans ce sens, elle juge que l'annexe B de l'accord-cadre représente une bonne base pour les négociations à venir sur les modalités ${ }^{14}$. Pour la suite des négociations, elle met en avant la nécessité de s'assurer de la réduction non seulement des droits de douane mais également des barrières non tarifaires. La Suisse souligne également l'importance de l'amélioration substantielle de l'accès aux marchés des pays émergents. Au niveau des services, secteur également de haute importance, elle estime que de nombreux progrès devront encore être réalisés, notamment en améliorant la qualité des offres. La Suisse appuyait fermement le lancement de négociations sur les quatre thèmes de Singapour. Elle déplore donc que l'investissement, la transparence des marchés publics et la politique de la concurrence n'aient pas été inclus dans les négociations du cycle de Doha. Elle se réjouit par contre de la décision d'y inclure la facilitation des échanges. Enfin, en ce qui concerne le processus de négociations, elle critique et met en garde contre la tenue de négociations à huis clos entre certains pays membres ${ }^{15}$, notamment dans le cadre de l'agriculture.

10 Notamment pendant la CNUCED XI à São Paulo en juin 2004.

11 Département fédéral de l'économie, La Suisse salue la nouvelle impulsion donnée à Genève aux négociations sur le cycle de Doha, communiqué de presse, $1^{\text {er }}$ août 2004, <www.seco.admin.ch> $>$ Politique économique extérieure >OMC.

12 Le G-10 se compose des pays suivants : Bulgarie, Taiwan, Islande, Israël, Liechtenstein, Japon, Corée, île Maurice, Norvège et Suisse.

13 Par exemple l'environnement, le développement rural ou la sécurité alimentaire.

14 WTO: Bericht über die Sitzung des Generalrates vom 31. July 2004 und die Annahme der Entscheide für eine Neustart der Doha-Runde, 1. August 2004, p. 3, <www.seco.admin.ch> >Politique économique extérieure $>\mathrm{OMC}$.

15 Les Etats-Unis, l'Union européenne, l'Australie, le Brésil et l'Inde se sont réunis plusieurs fois à huis clos (voir plus bas le paragraphe sur l'agriculture). 


\subsubsection{Les résultats obtenus dans l'accord-cadre de juillet 2004}

\section{$\square$ Présentation générale}

Les membres de l'OMC ont adopté le 1' ${ }^{\text {er }}$ août 2004 un accord-cadre, composé de deux sections. D’une part, la décision contient une partie générale qui couvre notamment les points suivants: l'agriculture, le coton, l'accès aux marchés pour les produits non agricoles, le développement, les services, la facilitation des échanges et les trois autres «questions de Singapour» ${ }^{16}$. Dans cette partie générale, le délai pour conclure le cycle de Doha, envisagé initialement au $1^{\mathrm{er}}$ janvier 2005, a été reporté à une date ultérieure et l'organisation de la $6^{\mathrm{e}}$ Conférence ministérielle a été prévue pour décembre 2005 à Hongkong.

D'autre part, cette décision comprend quatre annexes relatives à l'agriculture, à l'accès au marché pour les produits non agricoles, aux services et à la facilitation des échanges, qui traitent ces sujets plus en détail.

L'accord-cadre de juillet 2004 marque une étape importante du cycle de Doha pour les pays membres de l'OMC. En effet, il représente une bonne base pour la poursuite des négociations. Pour les pays membres, le maintien d'un système commercial multilatéral, comprenant un processus de règlement de différends, est considéré comme essentiel. Cependant, les enjeux diffèrent suivant les secteurs et les pays. L'agriculture représente un bon exemple pour illustrer les intérêts divergents. En effet, certains pays en développement, notamment ceux membres du G-20 ${ }^{17}$, ont un intérêt marqué pour la libéralisation de ce secteur, car ils estiment pouvoir en retirer des avantages substantiels. D'autres pays en développement, importateurs nets de nourriture, sont par contre opposés à une telle libéralisation. Pour l'Union européenne ou la Suisse, l'agriculture représente plutôt un intérêt défensif, en comparaison avec la libéralisation des autres secteurs, notamment des produits non agricoles ou de la propriété intellectuelle. Quant aux pays les moins avancés (PMA), la situation est plus délicate. En effet, bien qu'ils soient exempts de la majorité des obligations négociées ou à venir, ils ne sont plus en mesure d'augmenter leur protection tarifaire et leur soutien interne.

\section{$\square$ Les questions de développement}

Les questions relatives au développement sont traitées dans la partie générale de la décision de juillet 2004. En effet, celle-ci réaffirme que «les intérêts des pays en développement et des pays les moins avancés » doivent être mis au centre du Programme de travail de Doha ${ }^{18}$. Elle rappelle aussi la nécessité de rendre les dispositions du traitement spécial et différencié ${ }^{19}$ pour les pays en développement «plus précises, plus effectives et plus opérationnelles » ${ }^{20}$. Elle souligne,

16 Ces sujets sont mentionnés dans la partie générale où il est exposé qu'ils ne feront pas partie du programme de Doha.

17 Le G-20 est un groupe de pays en développement, tels que le Brésil, l'Inde ou l'Afrique du Sud, qui prône une libéralisation de l'agriculture. Ce groupe s'était formé juste avant la Conférence ministérielle de Cancún en réponse aux positions de l'Union européenne et des Etats-Unis.

18 OMC, Programme de travail de Doha. Décision adoptée par le Conseil général le $1^{\text {er }}$ août 2004, 2004, doc. WT/L/579, par 1.d, p. 2.

19 Les dispositions relatives au traitement spécial et différencié, incluses dans les accords de l'OMC, donnent des droits spéciaux aux PVD et permettent aux pays développés de leur accorder un traitement plus favorable qu'aux autres membres de l'OMC.

20 OMC, Programme de travail de Doha. Décision adoptée par le Conseil général le 1er août 2004, op. cit., par. 1.d., p. 2. 
une fois de plus, l'importance de l'assistance technique et du renforcement des capacités en faveur des pays en développement pour notamment ajuster et diversifier leurs économies. La décision de juillet 2004 rappelle également que les préoccupations des pays les moins avancés devront être prises en compte dans les négociations. Elle réaffirme l'importance de trouver des solutions relatives aux problèmes, rencontrés par certains pays membres, au niveau de la mise en œuvre des accords de l'OMC. Enfin, cette décision recommande la prise en compte dans les négociations des questions relatives notamment à la sécurité alimentaire, aux préférences, aux produits de base et aux importations nettes de produits alimentaires. Elle préconise également de tenir compte des préoccupations des petites économies vulnérables, «sans que ne soit créée une sous-catégorie de membres $»^{21}$.

\section{$\square$ L'agriculture}

Le «Cadre pour l'établissement de modalités concernant l'agriculture» (ou Annexe $\mathrm{A})^{22}$ représente un compromis résultant de discussions longues et difficiles. Lors de ces discussions, le G-20 s'est confirmé comme un acteur important. Mais c'est surtout l'émergence du groupe des cinq parties intéressées qui est à relever, particulièrement parce qu'il a favorisé la conclusion de l'accordcadre de juillet. En effet, les Etats-Unis, l'UE, l'Australie, le Brésil et l'Inde se sont réunis à plusieurs reprises à huis clos et se sont mis d'accord sur un document, utilisé comme base du texte final. Ce rôle particulier n'a pas été au goût de certains autres pays membres qui se sont inquiétés de ce processus de négociations exclusif ${ }^{23}$. Cela d'autant plus que l'agriculture a été l'un des thèmes essentiels des négociations conduisant à l'ensemble des résultats de juillet. L'Annexe A traite des trois piliers de l'agriculture: le soutien interne, la concurrence à l'exportation et l'accès aux marchés. Il convient de relever que les pays les moins avancés ne sont pas obligés de prendre des engagements de réduction de leur soutien interne, de leurs subventions à l'exportation et de leurs tarifs ${ }^{24}$.

En ce qui concerne le soutien interne, l'accord-cadre contient des objectifs concrets de réduction au niveau global. De plus, un plafonnement à $5 \%$ de la production agricole des aides accordées dans le cadre de la catégorie bleue a été décidé. L'accord-cadre prévoit également la révision des critères de la catégorie verte, afin qu'elle n'aie réellement qu'une incidence tout au plus minimale sur les échanges ${ }^{25}$.

Par rapport à la concurrence à l'exportation, l'accord préconise clairement l'élimination de toutes les formes de subventions à l'exportation et des disciplines

21 Ibid., p. 3.

22 Ibid., pp. A-1 - A-9.

23 Notamment le G-10, dont la Suisse fait partie, qui regroupe des pays importateurs nets de nourriture et qui défend les aspects non commerciaux de l'agriculture.

24 OMC, Programme de travail de Doha. Décision adoptée par le Conseil général le $1^{\text {er }}$ août 2004, op. cit., Annexe A, par. 45, p. A-8.

25 Les mesures de soutien interne sont classées en trois catégories de couleurs différentes - en fonction des distorsions qu'elles engendrent sur les échanges commerciaux. La catégorie orange contient les soutiens ayant une incidence sur la production et les échanges: ces mesures doivent être réduites. La catégorie bleue représente les soutiens qui obligent les agriculteurs à limiter leur production: ces mesures ne sont pas limitées. La catégorie verte symbolise les soutiens qui n'ont pas ou peu d'effets de distorsion: ces mesures sont autorisées. 
concernant toutes les mesures à l'exportation d'effet équivalent ${ }^{26}$. L'objectif de trouver «une date butoir crédible» pour cette élimination représente un élément nouveau, même si cette date reste encore à négocier.

Enfin, dans l'accès aux marchés, les pays membres se sont accordés pour une amélioration substantielle, basée sur «une formule étagée qui tienne compte de leurs structures tarifaires différentes ${ }^{27}$. Tous seront en mesure de désigner des produits dits «sensibles» qui pourraient faire l'objet d'un traitement distinct. Les pays en développement auront une marge de manœuvre supplémentaire grâce à la possibilité de désigner un certain nombre de «produits spéciaux » qui pourront être soumis à un régime plus flexible.

\section{$\square$ L'accès aux marchés pour les produits non agricoles}

Le cadre pour l'établissement de modalités concernant l'accès aux marchés pour les produits non agricoles (ou Annexe B) est basé en grande partie sur le texte «Derbez», un projet de déclaration ministérielle sorti pendant la Conférence ministérielle de Cancún en septembre 2003, mais qui n'a pas été adopté. Cependant, pour tenir compte des revendications de plusieurs pays en développement, un certain nombre d'éléments ont été ajoutés à ce texte. En effet, dans l'accordcadre de juillet, le premier paragraphe, inexistant dans le texte initial, précise que des négociations additionnelles seront «nécessaires pour parvenir à un accord sur les détails spécifiques» contenus dans ce cadre, tels que la formule de réduction tarifaire ou la flexibilité pour les pays en développement. D'autre part, ceux-ci ne devront pas appliquer une réciprocité totale sur leurs engagements de réduction. Enfin, les pays les moins avancés seront exempts d'appliquer la formule tarifaire ${ }^{28}$.

\section{$\square$ Les services}

Annuaire 2004, n 1, chap. 6, point 6.2, voir «Thèmes de négociation», p. 93.

Les négociations sur les services sont basées essentiellement sur l'échange d'offres et de demandes de la part des pays membres. La décision de juillet 2004 contient des recommandations du Conseil du commerce des services (Annexe C) pour la poursuite des négociations ${ }^{29}$. Cette décision exhorte notamment les membres qui ne l'ont pas encore fait à présenter leurs offres initiales le plus rapidement possible. L'intérêt des pays membres, particulièrement ceux en développement, pour le mode $4^{30}$ a également été souligné. Dans la partie générale de la décision de juillet, le Conseil général propose l'échéance de mai 2005 pour la présentation des offres révisées.

26 Ce sont non seulement les subventions à l'exportation qui sont visées dans cet accord, mais également les crédits à l'exportation, les monopoles d'exportation des entreprises commerciales d'Etat et l'aide alimentaire.

27 Les détails de cette formule étagée restent encore à négocier, notamment selon les principes suivants : les droits de douane seraient divisés en différents groupes selon leur niveau respectif de droits consolidés et les droits de douane les plus élevés enregistreraient des réductions plus fortes.

28 Ce dernier point était déjà présent dans le texte de «Derbez».

29 Ces recommandations avaient été adoptées par le Conseil du commerce des services. Voir Conseil du commerce des services, Rapport du président au Comité des négociations commerciales du 7 juillet 2004, Session extraordinaire du Conseil du Commerce des Services, doc. TN/S/16, <www.wto.org>.

30 Le mode 4 concerne les personnes provenant d'un pays membre pour fournir un service dans un autre pays membre. 


\section{Facilitation des échanges}

La facilitation des échanges est le seul des quatre thèmes de Singapour à avoir été inclus dans la décision de juillet 2004. Les trois autres thèmes - l'investissement, la politique de la concurrence et la transparence des marchés publics - ont clairement été retirés des négociations du cycle de Doha ${ }^{31}$. Cette configuration répond aux craintes des pays en développement, fortement exprimées lors de la Conférence ministérielle de Cancún, face au lancement de négociations sur ces sujets.

Les négociations sur la facilitation des échanges se dérouleront au sein du «Groupe de négociation sur la facilitation des échanges» qui sera établi par le Comité des négociations commerciales. Elles viseront à «accélérer encore le mouvement, la mainlevée et le dédouanement des marchandises». Les modalités pour ces négociations (ou Annexe D) mettent clairement en avant la nécessité d'un traitement spécial et différencié ${ }^{32}$ et de l'octroi d'assistance technique pour les pays en développement et les pays les moins avancés.

\section{$\square$ Le coton}

ED Annuaire 2004, $\mathrm{n}^{\circ}$ 1, chap. 6, point 6.3, pp. 96-103.

En 2003, quatre pays d'Afrique de l'Ouest et du Centre (AOC), le Bénin, le Burkina Faso, le Mali et le Tchad, ont déposé une initiative sur le coton auprès de l'OMC. Cette initiative dénonçait les subventions octroyées par certains pays membres, notamment les Etats-Unis et l'Union européenne, et demandait leur élimination. La question du coton a figuré à l'agenda de la Conférence ministérielle de l'OMC qui s'est tenue à Cancún en septembre 2003. Puis, des négociations informelles ont été menées par les membres de l'OMC sur quatre sujets prioritaires, dont le coton ${ }^{33}$. Malgré le succès médiatique et le soutien de pratiquement tous les membres de l'OMC, la question du coton n'a que peu évolué lors des premiers mois de l'année 2004.

Au début de l'année 2004, les débats ont porté notamment sur l'intégration du coton au sein des négociations sur l'agriculture, soutenue par les Etats-Unis, l'Union européenne et le Secrétariat de l'OMC, ou sur son maintien en dehors du cadre agricole, soutenu par les pays africains. Suite à d'intenses négociations entre les Américains et les quatre pays de l'AOC, une issue a été trouvée. En effet, dans la décision du $1^{\text {er }}$ août 2004 du Conseil général de l'OMC, le coton a été intégré dans les négociations agricoles. Cependant, il convient de relever que le coton est le seul produit à disposer d'un paragraphe distinct non seulement dans la partie générale de la décision, mais également dans l'annexe relative à l'agriculture. De plus, dans cette annexe, il est précisé que le coton sera traité «de manière ambitieuse, rapide et spécifique $»^{34}$. Enfin, la question du coton sera abordée au sein d'un sous-comité du coton, établi en novembre 2004. Il importe de noter qu'à ce stade aucun engagement ferme n'a été émis par les

31 Il n'est pas précisé si ces thèmes sont également retirés de l'agenda général de l'OMC.

32 Les pays membres reconnaissent que dans le cadre de la facilitation des échanges, le traitement spécial et différencié devrait aller au-delà des périodes de transition pour la mise en œuvre des engagements.

33 Les trois autres sujets sont l'agriculture, l'accès aux marchés pour les produits non agricoles et les questions de Singapour.

34 OMC, Programme de travail de Doha. Décision adoptée par le Conseil général le $1^{e r}$ août 2004, op. cit., p. A-1. 
membres de l'OMC d'éliminer toutes les formes de subventions sur le coton. Les pays africains seront donc amenés à redoubler d'efforts pour matérialiser le potentiel de cette décision.

Il convient de relever que la demande initiale des pays africains d'obtenir une compensation financière transitoire n'est pas mentionnée dans la décision du $1^{\text {er }}$ août 2004. Elle a été dans les faits «remplacée» par le volet développement du texte approuvé par le Conseil général. Celui-ci invite instamment les pays développés à travailler sur les aspects développement du coton, que ce soit dans le cadre de leurs programmes bilatéraux ou sur le plan multilatéral. Des consultations seront par ailleurs engagées entre le directeur général de l'OMC et d'autres organisations internationales afin que les programmes existants ou à venir tiennent davantage compte des besoins de développement des pays dépendants du coton.

Finalement, la publication du rapport du groupe spécial de l'Organe de règlement des différends de l'OMC - dans le cadre du différend entre le Brésil et les Etats-Unis concernant le coton upland américain - représente un élément important pour les pays africains. En effet, ce rapport renforce les demandes des pays de l'AOC ${ }^{35}$ (voir plus bas l'encadré sur le règlement des différends).

\subsubsection{Réactions en Suisse aux résultats de juillet 2004}

Après l'échec de la Conférence de Cancún, les pays membres de l'OMC étaient presque dans l'obligation de parvenir à un accord-cadre pour éviter une crise du système multilatéral commercial. Même si certaines organisations non gouvernementales (ONG) avaient salué cette issue, nombreux étaient les observateurs qui soulignaient l'importance de continuer les négociations du cycle de Doha, particulièrement pour les pays en développement. Tous les acteurs concernés ont donc suivi avec intérêt les âpres négociations du premier semestre 2004 et leurs résultats de juillet.

La Fédération des entreprises suisses salue la conclusion de l'accord-cadre, car elle permet de relancer les négociations du cycle de Doha, essentielles pour l'économie suisse. Cependant, elle estime que cet accord ne représente qu'un «minimum absolu ${ }^{36}$. Elle regrette d'ailleurs que les résultats obtenus pour l'accès aux marchés pour les produits non agricoles et pour les services ne soient pas plus ambitieux. Elle déplore également que seule la facilitation des échanges, parmi les quatre thèmes de Singapour initiaux, ait été incorporée au cycle de Doha.

L'Union suisse des paysans (USP) regrette que l'accord-cadre de juillet 2004 ne prenne pratiquement pas en considération les aspects non commerciaux de l'agriculture. Elle estime que cet accord représente un enjeu énorme pour l'agriculture suisse, qui «devra consentir de gros sacrifices, sans que des domaines sensibles ne soient suffisamment pris en compte $»^{37}$. L'USP juge donc cet accord «déséquilibré».

35 Etats-Unis - Subventions concernant le coton upland. Rapport du Groupe spécial, doc. WT/DS267/R, 8 septembre 2004, <www.wto.org $>$.

36 Economiesuisse, Accord-cadre de l'OMC: un petit pas dans la bonne direction, communiqué de presse, $1^{\text {er }}$ août 2004.

37 Union suisse des paysans, Les enjeux de l'accord-cadre conclu à l'OMC sont énormes pour l'agriculture suisse, communiqué de presse, $1^{\text {er }}$ août 2004. 
Quant aux ONG suisses, elles considèrent cet accord-cadre également comme étant déséquilibré. En effet, la Déclaration de Berne dénonce les concessions élevées faites par les pays en développement, surtout dans les secteurs de l'accès aux marchés pour les produits non agricoles et les services ${ }^{38}$. En contrepartie, elle estime qu'ils n'ont obtenu que la promesse des pays industrialisés - sans date précise - de libéraliser leur agriculture. Cette critique est partagée par des ONG étrangères, telles qu'Oxfam International. De nombreuses voix, notamment celle de la Communauté de travail des œuvres d'entraide, se sont élevées également contre le manque de transparence dans le processus de négociations ${ }^{39}$.

\subsubsection{Prochaines étapes}

L'accord-cadre de juillet a été le point culminant de l'année 2004. Après l'été, les réunions ont repris à l'OMC, notamment concernant les discussions techniques. De nombreuses questions restent à préciser, particulièrement en termes de modalités ou de chiffres précis, pour que les négociations puissent aboutir. En effet, les pays membres, pressés par la nécessité de se mettre d'accord avant l'été, ont laissé de côté certains éléments difficiles.

La date qui devait marquer la fin du cycle de Doha, le $1^{\text {er }}$ janvier 2005, a été reportée, sans autre précision. Pour l'instant, les pays membres ne préfèrent pas se donner de date butoir pour la conclusion de ce cycle. Ils ont désormais en ligne de mire la prochaine étape cruciale, la Conférence ministérielle de Hongkong qui aura lieu en décembre 2005. Ils devront travailler encore davantage pour pouvoir obtenir des résultats concrets d'ici cette échéance.

\subsubsection{Accessions à I'OMC}

En date respectivement du 23 avril et du 13 octobre 2004, le Népal et le Cambodge sont officiellement devenus les $147^{\mathrm{e}}$ et $148^{\mathrm{e}}$ membres de $1^{\prime} \mathrm{OMC}$. Ces deux pays sont les premiers pays les moins avancés à entrer dans l'organisation en ayant suivi un réel processus d'accession.

메 Annuaire 2004, $\mathrm{n}^{\circ}$ 1, chap. 6, point 6.2, pp. 95-96.

Lors de la réunion du Conseil général du 27 juillet 2004, les pays membres ont convenu de lancer le processus d'accession pour la Libye. Cette dernière avait soumis sa demande d'accession en 2001. Celle-ci avait jusqu'ici toujours été refusée par les Etats-Unis, pour des raisons politiques ${ }^{40}$. La procédure officielle d'un candidat à l'accession débute par la création d'un groupe de travail par le Conseil général ${ }^{41}$, qui examine ses politiques commerciale et économique. Le pays candidat doit ensuite engager des négociations bilatérales avec chaque pays membre qui le requiert. Ces deux étapes terminées, le groupe de travail

38 Déclaration de Berne, OMC: les pays en développement paient le prix fort, communiqué de presse, 2 août 2004.

39 Communauté de travail des œuvres d'entraide, «La face est sauvée, mais l'avenir très incertain », Global $+, \mathrm{n}^{\mathrm{o}} 13,2004$.

40 C'est notamment l'abandon par la Libye de ses programmes d'armes de destruction massive qui a permis ce changement d'attitude des Etats-Unis. Voir U.S. Eases Economic Embargo against Libya, Statement of the Press Secretary, 23 April 2004, <www.whitehouse.gov> >News >News by Date $>$ April 2004.

41 Le groupe de travail est ouvert à tous les pays membres de l'OMC. 
prépare un projet de traité d'accession qui doit être accepté par les deux tiers des pays membres au minimum ${ }^{42}$. La durée du processus d'accession peut durer jusqu'à quinze ans, comme cela a été le cas de la Chine et du Népal. La requête de l'Iran d'accéder à l'OMC a par contre été à nouveau refusée par les Etats-Unis.

En mai 2004, la Fédération de Russie a conclu avec l'Union européenne (UE) ses négociations bilatérales relatives à son accession à l' $\mathrm{OMC}^{43}$. Les deux partenaires ont notamment réussi à s'entendre sur la politique énergétique russe en matière de prix, l'un des plus grands obstacles à un accord. Cet accord avec l'UE a grandement rapproché la Fédération de Russie de son entrée à l'OMC. Cependant, elle doit encore négocier des accords bilatéraux, notamment avec la Chine et les Etats-Unis. La Fédération de Russie n'envisage pas d'obtenir son ticket d'entrée à l'OMC avant début 2006.

Les groupes de travail pour l'Irak et l'Afghanistan ont été établis en décembre 2004 par le Conseil général. De nombreux observateurs estiment que l'Arabie Saoudite, le Liban, l'Ukraine et le Vietnam notamment sont sur la bonne voie pour accéder à l'OMC en 2005 ou au début 2006.

\section{Le règlement des différends à I'OMCa}

Deux cas d'une importance fondamentale, en particulier pour les pays en développement, ont fait l'objet d'un règlement de différend, dont le rapport est sorti en 2004: d'une part le cas du sucre, de l'autre celui du coton.

L'OMC est une organisation internationale composée de trois piliers. C'est en premier lieu un cadre de négociation commerciale, elle regroupe également l'ensemble des règles commerciales et, enfin, elle règle les différends entre pays membres. La procédure de règlement des différends est utilisée lorsqu'un ou plusieurs pays membres jugent qu'un autre pays membre enfreint les règles commerciales. Un mémorandumb établit un processus composé d'étapes qui sont soumises à des délaisc ${ }^{c}$.

1. Les parties au différend doivent d'abord essayer de s'entendre lors de consultations qui peuvent durer jusqu'à soixante jours.

2. Si ces consultations n'aboutissent pas, la partie plaignante peut demande l'établissement d'un groupe spécial. C'est I'Organe de règlement des différends $(\mathrm{ORD})^{d}$ qui est compétent pour l'établir, dans un délai de quarante-cinq jours. Ce groupe spécial a six mois pour terminer ses travaux et les présenter pour examen à I'ORD. Celui-ci ne peut les rejeter que par consensus.

3. Les travaux du groupe spécial, qui ne doivent pas dépasser les six mois, sont soumis à une procédure détaillée contenue dans le Mémorandum d'accord. Les deux parties présentent leurs arguments, qui sont suivis de leurs réfutations respectives. Si nécessaire, des experts peuvent être consultés pour des questions techniques. Le groupe spécial soumet ensuite son rapport intérimaire, qui peut être réexaminé dans un délai de deux semaines. Finalement, le rapport final est transmis aux deux parties. Sa distribution à tous les membres de I'OMC intervient dans les trois semaines. Le rapport devient une décision ou une recommandation de I'ORD dans les soixante jours suivants, sauf rejet par consensus.

4. Chaque partie peut faire appe/ de la décision, qui sera traité par un organe d'appel. La durée de la procédure ne doit pas dépasser les soixante jours. L'ORD a ensuite trente jours pour accepter ou rejeter le rapport de l'organe d'appel.

42 Pour en savoir plus sur l'accession, se référer au site de l'OMC, <www.wto.org > > Domaines $>$ Accessions.

43 Le processus d'accession de la Fédération de Russie a débuté en 1993. 
5. Si le pays visé par la plainte ne met pas en œuvre les recommandations du rapport du groupe spécial ou de l'Organe de règlement des différends, et qu'aucune compensation satisfaisante n'a pu être négociée entre les deux parties, la partie plaignante peut imposer à la partie adverse des sanctions commerciales limitées, sur autorisation de I'ORD.

En ce qui concerne le coton, le rapport final du groupe spécial sur le coton a été publié en date du 8 septembre 2004'. La plainte avait été déposée par le Brésil contre les Etats-Unis le 3 octobre 2002. Le Brésil soutenait que certaines des mesures de soutien interne américaines à leurs producteurs de coton étaient illégales et qu'elles lui portaient préjudice. Même s'il ne condamne pas toutes les subventions américaines mises en cause par le Brésil, le rapport du groupe spécial a donné en grande partie raison aux revendications brésiliennes. II a également invité les Etats-Unis à remédier à la situation. Ceux-ci ont fait appel de cette décision en date du 18 octobre 2004.

Le rapport final du groupe spécial sur le sucre a quant à lui été publié en date du 15 octobre 2004. Une plainte avait été déposée contre l'Union européenne par le Brésil et l'Australie le 1er octobre 2002 et par la Thaïlande le 20 mars $2003^{f}$. Les plaignants soutenaient que I'UE subventionnait ses exportations de sucre au-delà du niveau formellement notifié à I'OMC et donc violait l'accord sur l'agriculture de I'OMC. Le rapport du groupe spécial sur le sucre a donné raison aux parties plaignantes en concluant que les subventions de I'UE étaient non conformes aux règles de I'OMC. II a invité l'UE à se conformer à ses obligations. L'UE a annoncé son intention de faire appel de cette décision.

a Pour en savoir plus sur le règlement des différends de I'OMC: Secrétariat de I'OMC, Guide sur le système de règlement des différends de I'OMC, Montréal, Editions Yvon Blais, 2004; idem, Les procédures de règlement des différends de I'OMC, Montréal, Editions Yvon Blais, 2004 (2édition).

b Mémorandum d'accord relatif aux règles et procédures régissant le règlement des différends, «www.wto.org> $>$ Domaines $>$ Règlement des différends.

c Ces délais sont approximatifs.

d L'Organe de règlement des différends est composé de tous les membres de l'OMC. II exerce les pouvoirs du Conseil général et des conseils et comités des accords visés.

e Etats-Unis - Subventions concernant le coton upland. Rapport du Groupe spécial, doc. WT/DS267/R, 8 septembre 2004, $<$ www.wto.org $>$.

f Communautés européennes - Subventions à l'exportation de sucre. Plainte de l'Australie. Rapport du Groupe spécial, doc. WT/DS265/R; Plainte du Brésil, doc. WT/DS266/R; Plainte de la Thaïlande, doc. WT/DS283/R, 15 octobre 2004, $<$ www.wto.org $>$.

\subsection{Conférence des Nations unies sur le commerce et le développement (CNUCED)}

\section{Présentation de la CNUCED}

Etablie en 1964, la Conférence des Nations unies sur le commerce et le développement (CNUCED) a été créée pour répondre aux préoccupations des pays en développement. C'est le principal organe de l'Organisation des Nations unies qui traite des problèmes de commerce et développement. Son instance suprême est la Conférence des Etats membres, qui se tient tous les quatre ans. Cette dernière se penche sur les questions liées au commerce et au développement, et favorise la formation de consensus en la matière. D'autre part, elle permet la définition du rôle et des priorités de la CNUCED. Le suivi entre les conférences est assuré par le Conseil du commerce et du développement, qui supervise les activités de la CNUCED. II compte trois commissions qui traitent des questions de politique générale et orientent les travaux du Secrétariata. Celui-ci est lui-même composé d'un secrétaire général, d'un secrétaire général adjoint et de cinq divisions ${ }^{b}$. En 2004, la CNUCED comptait 192 pays membres et 400 employés; son budget s'élevait à 69 millions de dollars. Le siège de la CNUCED se trouve à Genève. La Suisse est membre de cette organisation depuis sa fondation et représente l'un des principaux donateurs en termes de coopération technique.

Le mandat de la CNUCED est d'aider les pays en développement à s'intégrer dans l'économie mondiale, tout en favorisant leur développement. Pour ce faire, elle a trois fonctions: 
$1^{0}$ elle tient le rôle de forum intergouvernemental de délibérations qui permet l'établissement de consensus:

$2^{\circ}$ elle entreprend des travaux de recherche, des analyses et la collecte de données;

$3^{\circ}$ elle fournit une assistance technique aux pays en développement.

a Les trois commissions sont la Commission du commerce des biens et services et des produits de base, la Commission de l'investissement, de la technologie et des questions financières connexes, et la Commission des entreprises, de la facilitation du commerce et du développement. De plus, il faut tenir compte de la Commission de la science et de la technologie au service du développement, qui est desservie par le Secrétariat de la CNUCED et est un organe subsidiaire du Conseil économique et social de l'ONU.

b Quatre divisions se consacrent à des travaux de recherche et des activités d'assistance technique: la Division de la mondialisation et des stratégies de développement, la Division du commerce des biens et services et des produits de base, la Division de l'investissement, de la technologie et du développement des entreprises et la Division de l'infrastructure des services pour le développement et de l'efficacité commerciale; la cinquième division se consacre à la gestion. S'y ajoute un Programme spécial pour les pays en développement les moins avancés, sans littoral ou insulaires.

\subsubsection{1e Conférence à São Paulo, 13-18 juin 2004}

En 2004, la CNUCED a célébré son quarantième anniversaire en même tant qu'elle tenait, du 13 au 18 juin, sa $11^{\mathrm{e}}$ Conférence quadriennale à São Paulo, au Brésil. La CNUCED XI s'est donné comme sujet principal la cohérence entre les visions nationales et internationales du commerce et du développement pour favoriser la croissance économique et le développement. Quatre thèmes subsidiaires se trouvaient également à l'ordre du jour de ses travaux: les stratégies de développement dans une économie internationale mondialisée, le renforcement des capacités productives et de la compétitivité internationale, la contribution du système commercial international et des négociations commerciales au développement et le partenariat pour le développement. La CNUCED XI a abouti à l'adoption de trois documents: une déclaration intitulée L'esprit de São Paulo, un document négocié intitulé Le consensus de São Paulo ${ }^{44}$ et une déclaration sur le système global de préférences commerciales entre pays en développement ${ }^{45}$.

\section{$\square$ Cohérence entre les efforts nationaux et internationaux pour promouvoir le développement}

Le Consensus de São Paulo reconnaît que la globalisation peut représenter une forte source de croissance et de développement, tout en précisant que certains pays, notamment les pays les moins avancés, n'ont pas pu en tirer profit. Il convient donc de mettre au point des stratégies de développement qui tiennent compte de l'impact de la globalisation sur les dimensions humaines et sociales, afin de minimiser ses effets négatifs et de maximiser ses effets positifs. Ainsi, pour appuyer un processus de développement satisfaisant, il est essentiel de tenir compte de la responsabilité complémentaire du marché, d'une part, et de l'Etat, d'autre part. Le consensus souligne notamment le rôle de l'Etat en introduisant la notion d' «espace politique» (policy space), c'est-à-dire sa marge de manœuvre dans les politique nationales, notamment dans les secteurs du com-

44 CNUCED, Consensus de São Paulo et L'esprit de São Paulo, doc. TD/L.382 et TD/410, 2004, $<$ www.unctadxi.org $>$.

45 UNCTAD, Developing Countries Launch New Round of Trade Negotiations, press release, 17 June 2004, doc. unctad/press/pr/spa/2004/010 (y compris «The Sao Paulo Declaration on the Launching of the Third Round of Negotiations within the Global System of Trade Preferences among Developing Countries »), <www.unctad.org $>$. 
merce, de l'investissement et du développement industriel. Chaque gouvernement doit trouver un équilibre entre les avantages des règles et des engagements internationaux et les contraintes de la perte de cet «espace politique ${ }^{46}$. Cela est d'autant plus vrai que selon le consensus, il n'existe pas d'approche uniforme applicable à tous en termes de développement.

\section{$\square$ Cohérence entre les différentes institutions internationales}

Le Consensus de São Paulo souligne aussi l'importance d'une collaboration accrue entre les différentes activités de l'ONU. Une coopération étroite entre les organisations internationales financière, monétaires et commerciales est également encouragée, pour améliorer notamment les synergies, la complémentarité et le soutien mutuel. Le développement doit d'ailleurs se trouver au centre de leurs préoccupations. Cette cohérence entre les différentes institutions représente un élément essentiel pour créer un environnement international approprié qui soit au service de l'intégration des pays en développement et en transition dans l'économie mondiale ${ }^{47}$. La Suisse a d'ailleurs souligné l'importance de renforcer cette collaboration entre organisations, notamment entre la CNUCED et l'OMC, qui doivent rester complémentaires.

\section{$\square$ Promotion du commerce Sud-Sud}

Le secrétaire général de la CNUCED, Rubens Ricupero, a présenté les échanges commerciaux Sud-Sud comme l'un des thèmes majeurs de la CNUCED XI. Ces échanges s'élèvent déjà à $40 \%$ du commerce des pays en développement et représentent un potentiel énorme pour leur croissance ${ }^{48}$. Le Consensus de São Paulo encourage ces efforts et exhorte à les soutenir. Il souligne également l'importance de l'utilisation de préférences commerciales entre pays en développement. D'ailleurs, le troisième document d'importance issu de la CNUCED XI, la déclaration sur le système global de préférences commerciales entre pays en développement ${ }^{49}$, lance le troisième cycle de négociations commerciales entre pays en développement.

\section{$\square$ Rôle et mandat de la CNUCED}

Le rôle et le mandat de la CNUCED ont également été vigoureusement discutés. En effet, certains pays développés ont proposé de les limiter à l'octroi d'assistance technique au niveau national. Quant aux pays en développement, ils ont insisté pour un rôle plus ambitieux de la CNUCED, qui inclurait notamment la recherche et l'élaboration d'analyses politiques. Finalement, le Consensus de São Paulo insiste sur la responsabilité de la CNUCED pour contribuer à atteindre les objectifs internationaux de développement, notamment ceux de la Déclaration du millénaire. Il précise que la CNUCED devrait continuer de poursuivre son mandat tant en ce qui concerne l'assistance technique que le travail d'analyse et d'identification d'options politique, aux niveaux national et global ${ }^{50}$.

46 CNUCED, Consensus de São Paulo, op. cit., par. 6, 7, 8,12 et 13.

47 Ibid., par. 10, 11 et 17.

48 United Nations Non-Governmental Liaison Service, Roundup 115, July 2004, p. 1, <www.un-ngls. org $>$.

49 Le système global de préférences commerciales a été établi en 1989. Ses pays signataires s'accordent mutuellement des préférences tarifaires et non tarifaires. Cet accord a été ratifié par 44 pays.

50 CNUCED, Consensus de São Paulo, op. cit., par. 26. 


\section{$\square$ Position de la Suisse}

La Suisse a suggéré que la CNUCED poursuive son processus de réforme pour qu'elle concentre son travail dans les domaines où elle «a un avantage comparatif $[\ldots]$ vis-à-vis d'autres organisations internationales ${ }^{51}$. Elle souhaitait que la CNUCED soit soumise à une réelle évaluation de fond pour identifier ses priorités et poursuivre ses réformes. La Suisse a donc regretté que les conclusions de la CNUCED XI n'aient pas donné un résultat plus contraignant dans ce domaine. En ce qui concerne la participation de la Suisse aux discussions, il convient de noter qu'elle a occupé la vice-présidence du Bureau de la Conférence. Cette fonction lui a permis de faciliter le consensus entre les pays membres et la résolution des questions relatives aux négociations des documents de la CNUCED XI.

\section{$\square$ Rapports de la CNUCED}

La CNUCED publie régulièrement des rapports, dont notamment celui sur le commerce et le développement et le Rapport sur les pays les moins avancés. Le Rapport sur le commerce et le développement 2004 souligne l'importance d'un véritable système multilatéral aux niveaux commercial et monétaire internationaux pour permettre aux pays en développement de tirer le meilleur parti de la mondialisation $^{52}$. Le Rapport 2004 sur les pays les moins avancés traite du lien entre le commerce international et la pauvreté dans les PMA ainsi que de la cohérence entre les politiques nationales et internationales pour faire du commerce un moyen de réduction de la pauvreté ${ }^{3}$.

\subsubsection{Réactions à la CNUCED XI}

Il convient d'abord de relever la tenue d'un forum par la société civile, en marge de la CNUCED XI. L'un des sujets mis en avant était l'importance du maintien de l'indépendance et du rôle de la CNUCED en matière d'analyses critiques et d'octroi de recommandations. En ce qui concerne les résultats de la conférence, la Communauté de travail des œuvres d'entraide en tire un bilan globalement positif, notamment grâce à la reconnaissance de l' «espace politique» par rapport aux règles commerciales internationales, reconnu pour «la première fois [...] dans une déclaration intergouvernementale». Elle souligne également le maintien de l'indépendance critique de la CNUCED. A ce sujet, elle avait dénoncé la position suisse qui cherchait à «limiter le mandat de l'organisation et à la museler» pour qu'elle ne travaille que sur les questions liées aux pays les plus pauvres et à l'assistance technique. La Communauté de travail des œuvres d'entraide salue également la stimulation du commerce Sud-Sud. Par contre, elle regrette que les pays en développement aient dû renoncer à ce que «la CNUCED élabore des lignes directrices sur la responsabilité sociale et environnementale des multinationales $»^{54}$.

51 seco, Onzième Conférence ministérielle de la CNUCED, São Paulo, 10 juin 2004, <www.secocooperation.ch $>>$ Coopération au développement $>$ Commerce $>$ Les points forts.

52 CNUCED, Rapport sur le commerce et le développement 2004, New York; Genève, ONU, 2004.

53 CNUCED, Rapport 2004 sur les pays les moins avancés, New York; Genève, ONU, 2004.

54 Communauté de travail des œuvres d'entraide, Victoires pour le Sud, s.d., <www.swisscoalition.ch> $>$ Thèmes >Commerce/OMC. 


\section{SOURCES}

\section{Organisations internationales}

Conférence des Nations Unies sur le commerce et le développement (CNUCED)/United Nations Conference on Trade and Development (UNCTAD)

Consensus de São Paulo, 2004, doc. TD/410.

L'esprit de São Paulo, 2004, doc. TD/L.382.

Rapport sur le commerce et le développement 2004, New York; Genève, ONU, 2004, pp. 43-49.

Rapport 2004 sur les pays les moins avancés, New York; Genève, ONU, 2004.

Communiqué de presse

Developing Countries Launch New Round of Trade Negotiations, 17 June 2004, doc. unctad/ press/pr/spa/2004/010 (y compris «The Sao Paulo Declaration on the Launching of the Third Round of Negotiations within the Global System of Trade Preferences among Developing Countries»).

Organisation mondiale du commerce (OMC)/World Trade Organization (WTO)

Rapport sur le commerce mondial 2004, Genève.

Statistiques du commerce international 2004, Genève.

Communautés européennes - Subventions à l'exportation de sucre. Plainte de l'Australie. Rapport du Groupe spécial, doc. WT/DS265/R; Plainte du Brésil, doc. WT/DS266/R; Plainte de la Thälande, doc. WT/DS283/R, 15 octobre 2004.

Conseil du commerce des services, Rapport du président au Comité des négociations commerciales du 7 juillet 2004, Session extraordinaire du Conseil du Commerce des Services, doc. TN/S/16.

Etats-Unis - Subventions concernant le coton upland. Rapport du Groupe spécial, doc. WT/DS267/R, 8 septembre 2004.

Mémorandum d'accord relatif aux règles et procédures régissant le règlement des différends.

Programme de travail de Doha. Décision adoptée par le Conseil général le $1^{\text {er }}$ août 2004, 2004, doc. $\mathrm{WT} / \mathrm{L} / 579$.

Secrétariat de l'OMC, Guide sur le système de règlement des différends de l'OMC, Montréal, Editions Yvon Blais, 2004.

Secrétariat de l'OMC, Les procédures de règlement des différends de l'OMC, Montréal, Editions Yvon Blais, 2004 (2 édition).

Déclarations du président du Conseil général et du directeur général du 14 octobre 2003.

Remarques finales du président du Conseil général lors de la réunion du Conseil général des 15 et 16 décembre 2003.

Statement by Director-General of 15th December 2003.

Statement by the Chairperson of the General Council of 15th December 2003.

\section{Organisations non gouvernementales et groupes de pression}

Communauté de travail des œuvres d'entraide, Global +, nº 1, 2004.

Communauté de travail des œuvres d'entraide, Global $+, \mathrm{n}^{\circ} 13,2004$.

Déclaration de Berne, OMC: les pays en développement paient le prix fort, communiqué de presse, 2 août 2004.

Economiesuisse, Accord-cadre de l'OMC: un petit pas dans la bonne direction, communiqué de presse, $1^{\text {er }}$ août 2004.

Union suisse des paysans, Les enjeux de l'accord-cadre conclu à l'OMC sont énormes pour l'agriculture suisse, communiqué de presse, $1^{\text {er }}$ août 2004.

United Nations Non-Governmental Liaison Service, Roundup 115, July 2004.

\section{SITES INTERNET}

ACICI (Agence de coopération et d'information pour le commerce international) : <www.acici.org $>$.

ACWL (Centre consultatif sur la législation de l'OMC) : <www.acwl.ch $>$.

Banque mondiale: <www.worldbank.org $>$.

Communauté de travail des œuvres d'entraide: <www.swisscoalition.ch>.

CNUCED : <www.unctad.org $>$.

CNUCED XI : <www.unctadXI.org $>$.

Déclaration de Berne: <www.evb.ch>.

Fédération des entreprises suisses (economiesuisse) : <www.economiesuisse.ch $>$.

IDEAS Centre: <www.ideascentre.ch>.

ICTSD (International Centre for Trade and Sustainable Development) : <www.ictsd.org $>$. 
Maison-Blanche: <www.whitehouse.gov>.

Office of the United States Trade Representative: <www.ustr.gov>.

OMC: $<$ www.wto.org $>$.

Oxfam International : <www.oxfaminternational.org $>$.

ONU : <www.un.org >.

Secrétariat d'Etat à l'économie : <www.seco-admin.ch>.

South Centre: <www.southcentre.org $>$.

Union européenne: <http://europa.eu.int>.

Union suisse des paysans: <www.bauernverband.ch>.

United Nations Non-Governmental Liaison Service: <www.un-ngls.org>. 